\title{
Reparación por abordaje solo subcutáneo laparoscópico para hernia posincisional; reporte de caso
}

\author{
Laparoscopic subcutaneous approach repair for \\ postincisional hernia; case report \\ Yisvanth Pérez Ponce, ${ }^{*}$ Carlos Alberto Santana Pérez, ${ }^{\ddagger}$ Luis Ángel Muciño Pérez ${ }^{\ddagger}$ \\ * Cirugía y Medicina de Alta Especialidad Sepsis Obesidad y Cirugía Compleja, A.C. Especialidad de \\ Cirugía General, Universidad Autónoma del Estado de México (UAEMex). FACS. \\ ‡ Especialidad de Cirugía General. \\ Centro Médico ISSEMyM Toluca. México.
}

\section{RESUMEN}

Introducción: La hernia incisional es una complicación de la cirugía abdominal, definida como cualquier brecha en la pared abdominal con o sin una protuberancia en el área de una cicatriz posoperatoria perceptible o palpable mediante examen clínico o imagenología. Presentación del caso: Se presenta caso de una mujer de 34 años, quien es protocolizada por presentar hernia incisional subxifoidea, posterior a colecistectomía laparoscópica, encontrando un defecto herniario en región subxifoidea M3 según la clasificación europea de hernias con contenido de epiplón, sin compromiso vascular y hernia umbilical M3 con salida de epiplón, sin asa, demostrado por ultrasonido abdominal. Se decide someter a plastia de pared mediante abordaje de mínimo acceso asistido por laparoscopía, encontrando defecto supraaponeurótico epigástrico de $2 \mathrm{~cm}$ y defecto umbilical de $3 \mathrm{~cm}$. Se realiza cierre primario con puntos Smead Jones y se realiza plastia con técnica supraaponeurótica laparoscópica subcutánea (SCOLA). Resultados: Evolución sin complicaciones trans ni posoperatorias con egreso 24 horas posteriores al evento. Conclusiones: Este abordaje laparoscópico es una opción factible reproducible con baja morbilidad, para defectos en línea media con baja tasa de complicaciones y adecuado resultado estético.

Palabras clave: Hernia ventral, plastia ventral, laparoscópico, posincisional.

\section{ABSTRACT}

Introduction: Incisional hernia is a complication of abdominal surgery, defined as any gap in the abdominal wall with or without a protrusion in the area of a perceptible or palpable postoperative scar by clinical examination or image study. Case presentation: A case of a 34-year-old female patient is presented, who is studied for presenting a subxiphoid incisional hernia, after laparoscopic cholecystectomy, finding a hernia defect in the subxiphoid region M3 according to the European classification of omentum-containing hernias, without vascular compromise and M3 umbilical hernia with omentum outlet, without a intestinal loop, demonstrated by abdominal ultrasound. It was decided to undergo a wall plasty using a minimally invasive laparoscopic approach, finding a $2 \mathrm{~cm}$ epigastric supraponeurotic defect and a $3 \mathrm{~cm}$ umbilical defect. Primary closure was performed with Smead Jones sutures and plasty with subcutaneous laparoscopic supraponeurotic technique (SCOLA) with polipropilen mesh placement. Results: Follow up was uneventful without trans or post-operative complications and discharge 24 hours after the event. Conclusions: This laparoscopic approach is a reproducible and feasible option with low morbidity, for midline defects with a low complication rate and an adequate aesthetic result.

Keywords: Ventral hernia, ventral plasty, laparoscopic, postincisional.

Recibido: 05/06/2020. Aceptado: 24/08/2020.

Correspondencia: Carlos Alberto Santana Pérez

Centro Médico ISSEMyM Toluca «Lic. Arturo Montiel Rojas». Av. Baja Velocidad, San Jerónimo Chicahualco, Toluca, Estado de México. E-mail: casp.med@gmail.com

Citar como: Pérez PY, Santana PCA, Muciño PLÁ. Reparación por abordaje solo subcutáneo laparoscópico para hernia posincisional; reporte de caso. Rev Mex Cir Endoscop. 2020; 21 (4): 210-212. https://dx.doi.org/10.35366/101221 


\section{INTRODUCCIÓN}

La hernia incisional es una complicación frecuente de la cirugía abdominal, definida por Korenkov y colaboradores, como «cualquier brecha en la pared abdominal con o sin una protuberancia en el área de una cicatriz posoperatoria perceptible o palpable mediante examen clínico o imagenología». Existen distintos abordajes en la reparación de los defectos herniarios. Se ha encontrado beneficio en el proceso de recuperación al realizar técnica laparoscópica con base en la clasificación y tipo de hernia, ${ }^{1}$ como marca la clasificación de las hernias incisionales de Chevrel: ${ }^{2}$

M1: subxifoidea (desde el xifoides hasta $3 \mathrm{~cm}$ en sentido caudal).

M2: epigástrico (desde $3 \mathrm{~cm}$ por debajo del xifoides hasta $3 \mathrm{~cm}$ por encima del ombligo).

M3: umbilical (desde $3 \mathrm{~cm}$ arriba hasta $3 \mathrm{~cm}$ debajo del ombligo).

M4: infraumbilical (desde $3 \mathrm{~cm}$ por debajo del ombligo hasta $3 \mathrm{~cm}$ por encima del pubis).

M5: suprapúbico (desde hueso púbico hasta 3 cm cranealmente).

La tasa de complicaciones y éxito en comparación con la cirugía abierta ha demostrado actualmente ser mínima, y el resultado estético mucho mejor.,

\section{PRESENTACIÓN DEL CASO}

Se presenta caso de una mujer de 34 años, quien es protocolizada por presentar hernia incisional subxifoidea,

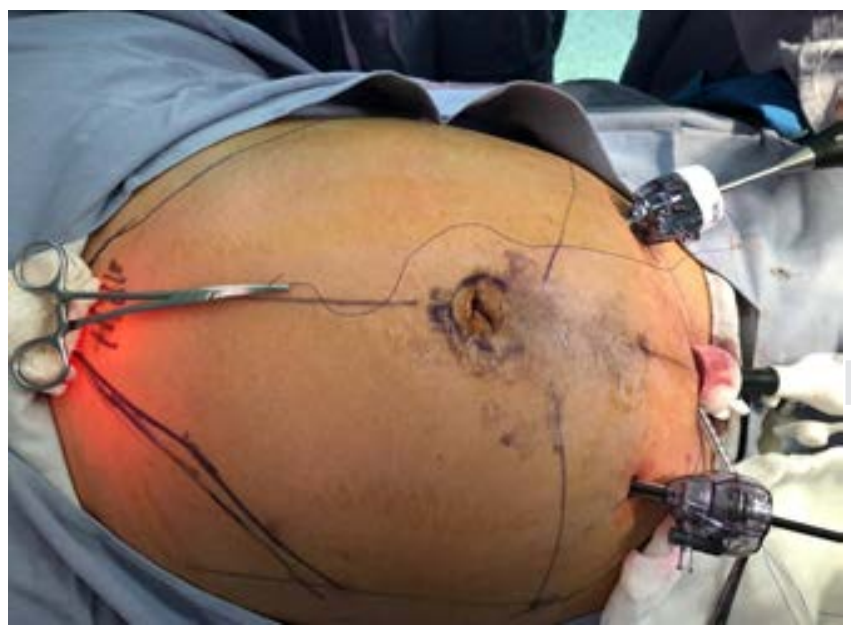

Figura 1: Posición del paciente y referencias anatómicas para la colocación de trocares en abordaje subcutáneo.

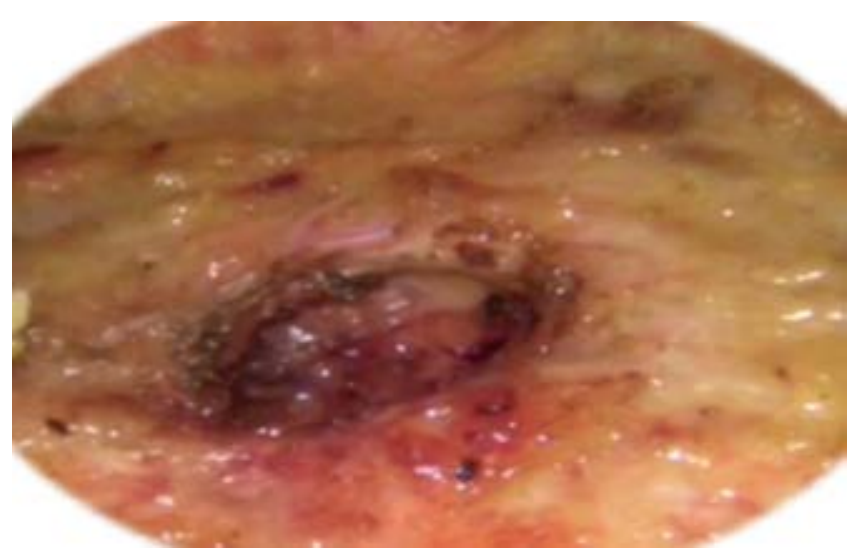

Figura 2: Defecto aponeurótico subxifoideo.

posterior a colecistectomía laparoscópica, encontrando un defecto herniario en región subxifoidea M3 según la clasificación europea de hernias con contenido de epiplón, sin compromiso vascular y hernia umbilical M3 con salida de epiplón, sin asa, demostrado por ultrasonido abdominal.

Se decide realizar un abordaje laparoscópico únicamente subcutáneo y colocación de material protésico de polipropileno, aceptando y firmando consentimiento informado. Se realizó tratamiento quirúrgico mínimamente invasivo asistido por laparoscopía, colocando trocares de trabajo de $10 \mathrm{~mm}$ suprapúbico y 2 de $5 \mathrm{~mm}$ en línea medioclavicular en cuadrante inferior derecho e izquierdo (Figura 1), encontrando defecto supraaponeurótico epigástrico de $2 \mathrm{~cm}$ y defecto umbilical de $3 \mathrm{~cm}$ (Figura 2). Se realizó cierre primario con puntos Smead Jones con sutura Monocryl ${ }^{\circledR}$ 2-0 (Figura 3) y se realizó plastia con técnica supraaponeurótica laparoscópica sólo subcutánea (SCOLA), con colocación de material protésico de polipropileno monofilamento (Figura 4). Se colocó Biovac ${ }^{\circledR}$ supraaponeurótico. Sin complicaciones transoperatorias, tiempo quirúrgico 60 min. Se egresó 48 horas después del evento quirúrgico, con adecuada evolución posquirúrgica, sin sangrado activo en los puertos laparoscópicos, no se palpan defectos aponeuróticos ni defectos herniarios a los seis meses del posoperatorio.

\section{DISCUSIÓN}

En la actualidad, existen diferentes tipos de abordajes para la reparación de defectos aponeuróticos primarios o posincisionales, que varían desde la técnica convencional abierta hasta los métodos de mínimo acceso. Si bien no existe una regla para la elección del mejor abordaje, esto dependerá de la selección adecuada del paciente, así como del momento y presentación clínica de la hernia, sin olvidar la experiencia del cirujano en la técnica a elegir. 


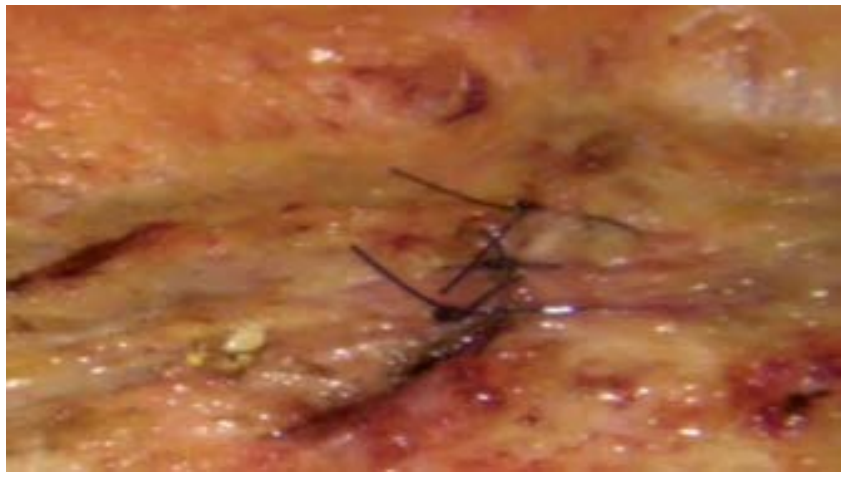

Figura 3: Cierre primario de defecto subxifoideo con material monofilamento no absorbible.

\section{CONCLUSIONES}

El abordaje de mínimo acceso asistido por laparoscopía para hernia ventral de línea media, con cierre primario y reforzamiento supraaponeurótico (onlay) con material protésico, es una opción viable, reproducible, factible de realizar, permite menor trauma a la pared abdominal, un menor tiempo de recuperación posoperatoria, menor cantidad en la ingesta de tratamiento analgésico, por lo que es una opción en tratamiento quirúrgico de pacientes con hernias incisionales.

\section{AGRADECIMIENTOS}

Expresamos al Centro Médico ISSEMyM Toluca y al Servicio de Cirugía General por su personal médico, enfermería

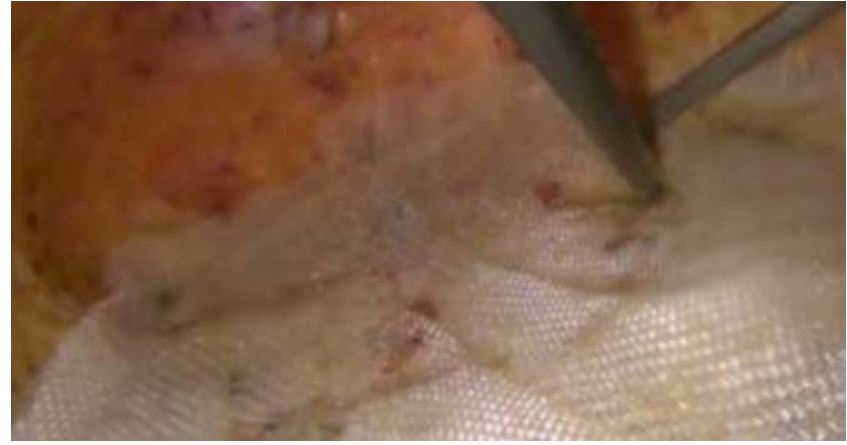

Figura 4: Colocación de malla de polipropileno. Nótese el adecuado espacio para su manipulación.

y logístico que se caracteriza por su entrega y dedicación a los pacientes.

\section{REFERENCIAS}

1. Korenkov M, Paul A, Sauerland S, Neugebauer E, Arndt $M$, Chevrel JP et al. Classification and surgical treatment of incisional hernia. Results of an experts' meeting. Langenbecks Arch Surg. 2001; 386: 65-73.

2. Rath AM, Chevrel JP. The healing of laparotomies: A bibliographic study Part two: Technical aspects. Hernia. 2000; 4: 41-48.

3. Muller-Riemenschneider F, Roll S, Friedrich M, Zieren J, Reinhold T, von der Schulenburg JM et al. Medical effectiveness and safety of conventional compared to laparoscopic incisional hernia repair: a systematic review. Surg Endosc. 2007; 21: 2127-2136.

4. Jonsson B, Zethraeus N. Costs and benefits of laparoscopic surgery--a review of the literature. Eur J Surg Suppl. 2000; 48-56. 